\title{
Improving the acute care of COPD patients across Gloucestershire: a quality improvement project.
}

\author{
Craig Miller, Claire Cushley, Kasey Redler, Claire Mitchell, Elizabeth Aynsley Day, Helen Mansfield, Abigail Nye \\ Gloucestershire Hospitals NHS Trust
}

\begin{abstract}
Admissions for exacerbations of chronic obstructive pulmonary disease (COPD) present a significant proportion of patients in the acute medical take. The British Thoracic Society (BTS) provides guidelines for time specific interventions, that should be delivered to those with an acute exacerbation of COPD through the admission care bundle. These include correct diagnosis, correct assessment of oxygenation, early administration of treatment, recognition of respiratory failure, and specialist review. Gloucestershire Hospitals NHS Foundation Trust (GHNHSFT) chose improvement in acute COPD care to be a local Commissioning for Quality and Innovation (CQUIN) scheme, which enables commissioners to reward excellence by linking a proportion of English healthcare providers' income to the achievement of local quality improvement goals. The effects of initiatives put in place by senior clinicians had waned, and further improvements were required to meet the CQUIN target.
\end{abstract}

The aim of the scheme was to improve compliance with the BTS guidelines and CQUIN scheme for patients admitted with an exacerbation of COPD.

Specific bundle paperwork to be used for all patients admitted to the Trust with an exacerbation of COPD was introduced to the Trust in June 2014, with training and education of medical staff at that time. This had improved compliance rates from 10\% to $63 \%$ by September 2014 . Compliance with each intervention was audited through the examination of notes of patients admitted with an exacerbation of COPD. Compliance rates had plateaued over the last three months, and so a focus group involving junior medical staff met in September 2014 to try to increase awareness further, in order to drive greater improvements in care, and meet the CQUIN requirements. Their strategies were implemented, and then compliance with the CQUIN requirements was reaudited as described above.

The December 2014 audit results showed a further improvement in overall COPD care, with $73 \%$ of patients receiving all elements of the COPD admission care bundle, versus $63 \%$ in September 2014. Appropriate blood gas analysis, nebuliser administration, and respiratory review also improved. Prescription of steroids and antibiotics remained static, with $96 \%$ of patients receiving these treatments within four hours in the emergency department (ED). The only criteria which showed a decline was appropriate oxygen prescription, which dipped from $97 \%$ to $87 \%$.

After the effect of initial strategies plateaued, this quality improvement project facilitated a further increase in compliance with the CQUIN targets, both improving patient care, and safeguarding continued CQUIN funding. There is further work to be done to maintain and support further improvement in standards, and to encourage use of the COPD admission bundle paperwork for documentation purposes.

\section{Problem}

Chronic obstructive pulmonary disease (COPD) is a common condition characterised by progressive respiratory airflow obstruction that is not fully reversible. Acute exacerbations of COPD are responsible for more than a million NHS bed days per annum, presenting a huge health burden.[1] Furthermore, exacerbations have a high mortality, approximately $10 \%$, and COPD is the fifth leading cause of death in the UK.[2] However, good acute medical care can improve outcomes, both in terms of the individual's mortality and morbidity, and the health burden to the NHS, ie number of bed days.[3]

The British Thoracic Society (BTS) have developed guidelines to improve the acute care of those patients admitted with an exacerbation of COPD.[4] The BTS COPD admission care bundle provides an outline for a group of time specific interventions that should be delivered to every patient admitted with an acute exacerbation of COPD, to optimise their initial management. It is focused on five key areas: correct diagnosis, correct assessment of oxygenation, early administration of treatment, recognition of respiratory failure, and specialist review.

In view of this, Gloucestershire Hospitals NHS Foundation Trust (GHNHSFT) had chosen improvement in acute COPD care to be a local Commissioning for Quality and Innovation (CQUIN) scheme. The national CQUIN payment framework "enables commissioners to reward excellence, by linking a proportion of English healthcare providers' income to the achievement of local quality improvement goals."[5]

\section{Background}


The Trust had previously identified the management of patients with an exacerbation of COPD could be improved, and this was highlighted by respiratory and emergency clinicians. The subsequent publication of the BTS COPD admission care bundle provided a framework for improvement. Linking acute COPD care to improvement commissioning through the CQUIN scheme helped motivate the Trust to support and invest in this quality improvement project.

The audit department at GHNHSFT began assessing compliance to the BTS COPD admission care bundle, and consequently created the COPD admission worksheet (see Appendix 1). This additional piece of paperwork is supplementary to the patient's main admission notes. It highlights each element of the care bundle, and can be completed as the patient progresses through their admission. Having a COPD care worksheet has two beneficial effects: firstly, to act as an aide mémoire for clinicians, and secondly, to facilitate efficient audit.

One year after the initial launch of the COPD care bundle with associated educational sessions, and emphasis with the unscheduled care and respiratory settings, compliance was found to have improved from $10 \%$ to $63 \%$. Results can be observed in Appendix 2. This showed that overall compliance with the COPD care bundle targets were generally good, however improvement had plateaued. We needed to find new ways of increasing the profile of the COPD worksheet, while educating clinicians and other staff to promote excellent patient care, and to drive for our CQUIN target of $70 \%$ compliance with all elements of the COPD care bundle for patients attending in December.

\section{Baseline measurement}

Initial compliance with each intervention of the COPD admission care bundle was measured through audit of 30 patients chosen at random from patients admitted with exacerbations of COPD, identified via our emergency department (ED) coding system. This sample size is consistent with all audits performed within our Trust for CQUIN projects. One year after the care bundle was introduced in June 2013 , compliance had increased from $10 \%$ to $60 \%$.

Compliance then plateaued, with rates in July 2014 of $60 \%$, August 2014 of 55\%, and September 2014 of 63\% (Appendix 2).

Compliance rates with the individual parameters of the COPD care bundle are shown in Appendix 3. This shows consistently high rates of oxygen administration, but variable levels of compliance with all other parameters. Of note was the respiratory review compliance, which was often below $80 \%$.

See supplementary file: ds6575.pdf - "Appendix 1. An example of the COPD worksheet used in the Emergency Department."

\section{Design}

In aiming to improve compliance, and thus patient care, we had to understand the barriers to completion of the care bundle. Therefore, all juniors working in the ED were invited to be involved in this quality improvement project. Volunteers formed a focus group to act alongside senior medical staff and Trust leads for patient safety, to develop new strategies to drive further improvement.

\section{Strategy}

PDSA cycle 1: The focus group met in September 2014. We noted that the small patient numbers audited could have a proportionally big impact on the percentage compliance, ie one patient not receiving all elements of the care bundle could drop compliance rates by three percent. It was agreed that trying to improve compliance with all individual parameters was important.

After brainstorming, several methods of increasing overall awareness of the respiratory bundle were agreed to try and improve compliance. Those methods implemented included:

- An information poster devised and displayed in the ED next to the blood gas machine, the acute medical admissions unit, and the staff bathrooms

\section{- Reminder emails to ED and acute medical unit staff}

- A teaching session at mandatory foundation doctor teaching

- Group members championing the completion of the full bundle criteria and paperwork during their own clinical practice.

Feedback received following these actions included the inability to locate the COPD bundle worksheet, lack of awareness of nursing staff with regard to the worksheet, and the time consumption of further paperwork.

PDSA cycle 2: A qualitative audit was also suggested and undertaken by the junior doctor team, scrutinising notes to explore the reasons targets were not met, and to identify further areas to best target improvement. The design of this audit entailed junior doctors dividing up a five day period from 22 September to 26 September 2014, and auditing compliance on a daily basis. The ED coding system was used to identify patients admitted the day before. ED and hospital notes were then reviewed as promptly as possible after the patient's admission. This often entailed a visit to the ward areas, in order to review patient notes. We hypothesised that this may reveal previously hidden barriers, such as demand and pressure in the ED at the time. This had a useful side effect of occasionally allowing us to talk to the clinicians who were treating the patients, and ask them about their awareness of the bundle, barriers to compliance, and facilitated a brief educational encounter. Unfortunately, due to clinical rota design, the appropriate clinician was not always available. Other than highlighting the issues already identified, this did reveal one interesting point: some patients were not being flagged to the respiratory team for specialist review, as the acute medical team were planning to discharge them that day. This meant that eligible patients were not able to receive their specialist 24 hour review, even though the specialty team was available to see them.

This feedback was discussed at a further focus group, and the 
following solutions devised:

- Inability to locate worksheet: clear folders containing the COPD worksheets were placed alongside the posters reminding clinicians about the care bundle, increasing availability and visual reminders in the department. Furthermore, a separate filing drawer was created to ensure a consistent location of the COPD worksheet.

- Lack of awareness of nursing staff: as nurses are a valuable part of the clinical team in unscheduled care, often guiding junior staff who frequently rotate through their department, prompting medical staff, and preparing the paperwork, improving their awareness would help highlight the care bundle to all. Communication was sent via weekly nursing departmental meetings.

\section{- Additional paperwork: the respiratory worksheet presented an additional piece of paperwork to complete in an already paperwork heavy environment. However, frustrations with this could be appeased through education around the reasons for and importance of completing the bundle. Although teaching of the bundle had happened within unscheduled care, we felt we needed to reach out to the wider junior doctor community who often rotated through unscheduled care, or might be caring for these patients when on call. We delivered a session at the regular Trust wide foundation program teaching.}

\begin{abstract}
- Patients not visible to the respiratory specialists: the respiratory consultant involved in the project liaised with the acute medical teams, re-emphasising the availability of the respiratory team, and the importance of flagging these patients to them through a "respiratory triage list" that was already in place.
\end{abstract}

In addition to these formal interventions, those involved continued to champion and educate the staff with whom they worked. This work took place during October to November 2014.

Of the interventions undertaken to improve compliance, there was discussion as to the expected impact and merit of each. Due to the short time frame over which we were trying to have an impact, these measures were all implemented simultaneously. Therefore it is difficult to quantify the impact of each strategy.

The rolling CQUIN audit was then analysed to assess for improvement.

\section{Results}

The effect of our intervention was measured, as described previously, by calculating the percentage of compliance within each element of the respiratory care bundle.

The CQUIN target was a compliance rate of $70 \%$ for all elements of the COPD bundle, for patients attending in the month of December 2014. Our strategy led to an improvement with compliance rates, increasing from 63\% for patients attending in September 2014 to 77\% in October 2014, and maintained at 77\% in November 2014. Compliance rates remained above the CQUIN target, at $73 \%$, for patients attending in December (Appendix 4).
Our interventions took place during the months of October and November 2014, when compliance rates were highest. However, it is interesting to see that compliance rates were sustained into the next quarter, with rates of $73 \%$ in January $2015,70 \%$ in February 2015, and 77\% in March 2015 (Appendix 5).

Analysing the individual compliance elements, appropriate blood gas analysis, nebuliser administration, and, of note, respiratory review all improved. Prescription of steroids and antibiotics remained static, with $96 \%$ of patients receiving these treatments within four hours in the ED. The only criteria which showed a decline was appropriate oxygen prescription, which dipped from $97 \%$ to $87 \%$. See Appendix 6 for these results in more detail. Surprisingly, despite the improvement in clinical parameters, the presence of the COPD admission bundle paperwork in patient notes at review remained at $37 \%$.

See supplementary file: ds6565.xlsx - "Appendix 2-6: COPD audit results"

\section{Lessons and limitations}

There were several limitations to our quality improvement project. These included relatively small numbers of eligible patients, especially in September; and a small sample size, which has the potential to distort data; however, we are limited by the number of cases that present to the ED.

A need for rapid improvement led to all strategies being introduced simultaneously; therefore, it is hard to evaluate the impact of each.

The COPD bundle is prescriptive in nature, and acts as an aide mémoire for clinicians caring for those with exacerbations of COPD; however, at times this could clash with other clinical priorities such as "Sepsis Six," meaning that choices about antibiotics were altered. Individual patient factors, such as patients who had already had a high dose of steroids from the GP, and palliative patients, meant that treatment was appropriately adjusted, but could affect the data. These patients could be excluded from the analysis, but this would further impact on the small sample size.

It seems the biggest improvement was seen when the effort put into marketing the care bundle was at its most intense. Raising its profile in daily conversation and grabbing people's attention by posters is difficult to maintain; through improving clinicians' fundamental awareness and understanding of the pathway, we produced a sustained effect.

The BTS clinical guidelines are held as a gold standard within the UK; however, this may not be true for other countries. What we have shown through this project is that whatever the clinical pathway you may be trying to implement, involving junior doctors can be a productive way of finding new avenues to utilise, to enhance compliance.

\section{Conclusion}




\section{BMJ Quality Improvement Reports}

Acute admissions for exacerbations of COPD represent a significant volume of the workload in the ED and acute medical unit. The COPD admission bundle used at GHNHSFT provides a framework for excellent care for this high risk cohort of patients, and is subject to CQUIN commissioning targets.

Involving the junior medical team with this quality improvement project helped identify barriers to further improvement, and produced strategies that would directly impact the group of medics using the care bundles. This facilitated a further increase in compliance with the CQUIN targets after the effect of earlier, senior, lead initiatives had waned, improving both patient care and safeguarding continued CQUIN funding. There is further work to be done to maintain and support further improvement in standards, and to encourage use of the COPD admission bundle paperwork for documentation purposes.

\section{References}

1. Hopkinson NS, Englebretsen C, Cooley N, et al. Designing and implementing a COPD discharge care bundle. Thorax 2012;67(1):90-2.

2. British Lung Foundation. Invisible lives: Chronic Obstructive Pulmonary Disease (COPD) - finding the missing millions. 2007. https://www.blf.org.uk/Files/94ff4ae1-1858-485fae85-a06200ded618/Invisible-Lives-report.pdf

3. National Institute for Health and Care Excellence. Chronic obstructive pulmonary disease in over 16s: diagnosis and management. NICE guidelines CG101. 2010.

http://www.nice.org.uk/guidance/cg101

4. Matthews $\mathrm{H}$, Tooley C, Nicholls C, Lindsey-Halls A. Care bundles reduce readmissions for COPD. Nursing Times 2013;109(7):18-20.

5. NHS Institute for Innovation and Improvement. Commissioning for Quality and Innovation (CQUIN) payment framework. http://www.institute.nhs.uk/commissioning/pct_portal/cquin.h $\underline{\mathrm{tml}}$

\section{Declaration of interests}

Nothing to declare

\section{Acknowledgements}

We would like to thank Jan Joseph in the audit department at Gloucestershire Hospitals NHS Foundation Trust, who kindly helped us collate relevant information, and Andrew Seaton, Director of Safety, for his help and guidance.

\section{Ethical approval}

According to the policy activities that constitute research at Gloucestershire Hospitals NHS Foundation Trust, this work met criteria for operational improvement activities exempt from ethics review. We used the following criteria for determining if improvement activities require ethics review. Policy criterion: The work is primarily intended to improve local care, not provide generalisable knowledge in a field of inquiry. We sought only to evaluate the improvements in compliance with the respiratory CQUIN as a result of auditing, and feedback of compliance rates to hospital staff. 